\title{
AIM: A personal view of where I have been and where we might be going
}

\author{
Alan Rector \\ Medical Informatics Group \\ Department of Computer Science \\ University of Manchester, Manchester M13 9PL, UK \\ Tel +44-161-275-6188/6239/7183 FAX +44-161-275-6204 \\ email rector@cs.man.ac.uk WEB www.cs.man.ac.uk/mig www.opengalen.org
}

Citation: Rector, AL (2001) AIM: A personal view of where I have been and where we might be going. Artificial Intelligence in Medicine 23:111-127

\begin{abstract}
My own career in Medical Informatics and AI in Medicine has oscillated between concerns with medical records and concerns with knowledge representation with decision support as a pivotal integrating issue. It has focused on using AI to organise information and reduce 'muddle' and improve the user interfaces to produce 'useful and usable systems' to help doctors with a 'humanly impossible task'. Increasingly knowledge representation and ontologies have become the fulcrum for orchestrating re-use of information and integration of systems. Encouragingly, the dilemma between computational tractability and expressiveness is lessening, and ontologies and description logics are joining the mainstream both in AI in Medicine and in Intelligent Information Management generally. It has been shown possible to scale up ontologies to meet medical needs, and increasingly ontologies are playing a key role in meeting the requirements to scale up the complexity of clinical systems to meet the ever increasing demands brought about by new emphasis on reduction of errors, clinical accountability, and the explosion of knowledge on the Web.
\end{abstract}

Keywords: Medical Records, Decision Support, Terminology, User Centred Design 


\title{
AIM: A personal view of where I have been and where we might be going
}

\author{
Alan Rector \\ Medical Informatics Group \\ Department of Computer Science \\ University of Manchester, Manchester M13 9PL, UK \\ Tel +44-161-275-6188/6239/7183 FAX +44-161-275-6204 \\ email rector@cs.man.ac.uk WEB www.cs.man.ac.uk/mig www.opengalen.org
}

\section{Early inspirations: Ledley and Lusted, Shortliffe, de Dombal, and Gorry}

Medical records and medical decision making have played alternating roles in my career and view of AI in medicine. (I would not presume to try give anything other than a highly personal retrospective as a 'portrait'.)

The most important interest, from early in medical school was Larry Weed and the Problem Oriented Medical Record [130]. It seemed to bring the hope of rationality to the buzzing and booming confusion facing an undergraduate medical student. It also seemed to promise what we would now call a 'safety culture' which seemed to a medical student aware of his own fallibility to be oddly lacking.

The first stirrings of interest in AI proper in medicine came from reading Feigenbaum's Computers and Thought [40] and Minsky and Papert's discouraging Perceptrons [77]. However, the symbolic and subsymbolic AI proposed there had first to make way for the more computationally attainable work applying the Bayesian methods pioneered by Ledley and Lusted [70].

Encountering Ledley and Lusted's work with the help of Eugene Ackerman and Laël Gatewod at University of Minnesota led directly to attempts to develop teaching routines which rated the appropriateness of tests based on maximum entropy criteria [98]. That work in turn led to a year's fellowship with Tim de Dombal in Leeds, whose work on abdominal pain and dyspepsia remains a model of careful clinical research[30-33, 135]. The concrete outcomes of the work with Tim de Dombal - applying Bayesian and other statistical techniques to the prognosis after surgery for carcinoma of the large bowel - are long and deservedly buried. However the experience of Tim's meticulous methods of data collection and emphasis on inter-rater reliability are lasting influences. (Indirectly it also led to my making my career in England).

Almost as important, Tim de Dombal introduced me to the Royal College of Physicians Computer Workshop which was a critical forum where statisticians and physicians met on equal terms. A profound impression from this period was of the frustration of the statisticians that the 'idiot Bayes' mechanisms usually outperformed their supposedly more sophisticated techniques. David Spiegelhalter was amongst the few statisticians to take the problem seriously - and succeed in solving it to produce 'well calibrated' techniques [120] and then to develop the algorithms which made Bayesian networks practical[29, 69]. Medical Informatics in general, and statistical decision making in particular owe a deep debt of gratitude to those who made that workshop possible, especially Wilfred Card and Robin Knill Jones of University of Glasgow.

However, statistical decision making was unsatisfying to me personally. Partly this was due to the difficulty experienced by Tim de Dombal in getting his work implemented. Rarely has a technique been more thoroughly evaluated; rarely has a technique been more consistently shown to improve patient care and reduce costs for minimal investment; rarely has it been less used.

My response was apparently not unique. Strangely, 'Medical Decision Making' and 'AI in Medicine' have usually gone their separate ways, at least in Europe, although the gap was partly bridged by a joint meeting of the European branches of the two societies in 1999.

In the meantime, I watched the major rule-based systems develop in the US with fascination. Shortliffe's Mycin [115] and perhaps even more Gorry, Pauker, and Kassier's work on knowledge representation [64, 91], as well as the other of the 'big four' from the AI in Medicine Workshop Abel[89, 90], Casnet[122], and Internist[76]. Perhaps most intriguing of all, Oncocin's attempt to integrate decision support into clinical care made a profound impression [57]. However, all were then beyond the aspirations of any group without access to very large computing resources - usually a DEC-10 running Interlisp.

My own efforts returned to medical records which at least offered the hope of letting doctors see what they were doing - something they clearly could not and did not except for short term results. 


\section{From Advising to Informing to Reducing Muddle}

This was the period of the 'AI winter' in the UK - a period when AI research was officially derided and nearly taboo with the research councils. That period ended with the panic caused by the Japanese announcement of their Fifth Generation Project which legitimised and stimulated an array of programmes in the UK as it did in many other countries. Our project was to look at general practitioners' decision making needs and to develop a series of decision support systems to meet them. In such days of optimism this was thought a reasonable goal. The most important part of the project were the psychological studies of decision making needs. Although never fully completed, they convinced me that question-and-answer advice systems were not the answer [18,100] and left me sceptical of 'expert systems' as practical clinical tools. At about this time Johnson and his colleagues published what is perhaps still the best ever field study of doctors' diagnostic reasoning [61-63], which further convinced me that what we were building were 'super-novice' rather than 'expert' systems, and that they were inappropriate for experienced clinicians.

The other key influence in this period was work on human error by Reason [94] and Rasmussen [92]. Those studies seemed to me to indicate that medicine really was a 'humanly impossible task' [58]. Examination of real medical records suggested that in even the best practices errors were common and frequently the result of not being able marshal the potentially available information. The existing systems, whether paper or computer based, left clinicians to make decisions in the dark - unable to see what they were doing - a view more in vogue today since the Institute of Medicine Report [67] than then.

The result was to shift our efforts from active advising to more subtle informing - and back to medical records, but from a new perspective. The direct outcome of the early decision support projects was the IMMEDIATE system [96] and an intense interest in human factors. These efforts developed into the PEN\&PAD programme.

PEN\&PAD's original goal was the User Centred Design of a 'useful and usable' system which would improve care in general practice. The project was based around a series of workshops with general practitioners simulating consultations with prototype systems and system components. The process was a fruitful partnership including clinicians, software developers, and psychologists. It was also enormous fun. It confronted me for the first time with just how great is the gulf in understanding between software developers and clinicians - in both directions [58,81,83] and gave promise of means to bring them together. (Unfortunately, such interdisciplinary work is expensive, and the circumstances which made it possible have been difficult to replicate).

Out of this period came many of the slogans which remain key to our group's activities.

- 'Shedding light' - clinicians will probably do the right thing if they can see what they are doing.

- 'Reducing muddle' - health care goes wrong because the multitude of people involved cannot possible manage and coordinate the total number of tasks

- 'There is no one way' - No one system will be right for everybody for everything. Searching for the 'best' way (and hence measures of central tendency) are largely a waste of time. A successful system will have to cope with a range of tastes and ways of working.

- 'The user is always right ..but the user is usually wrong' - the user is always right when they say they have a problem; they are usually wrong when they try to suggest a technical solution. Keep 'problem space' and 'solution space' separate.

- 'Swans paddle hard under water' - simplicity of use does not mean simplicity of design or implementation. Making a system seem natural to users is difficult and likely to require innovative software - otherwise why would most systems be so awkward?

- 'Medicine is big and complicated' - anything worthwhile to medicine as a whole must scale.

The forms-based user interface and navigation system which resulted was very different from either 'coding' or free text entry and allowed a remarkable amount of structured information to be recorded very quickly [65, 81, 83, 85]. Part of the system was eventually commercialised as Clinergy ${ }^{\mathrm{TM}}$, a front end for British General Practice systems [66].

However, PEN\&PAD was much more than just a user interface. It was a comprehensive attempt to produce a sound medical record system with as little compromise as the technology allowed:

- To be faithful - to provide a medical record model to faithfully represent the healthcare process, work that drew heavily on earlier work in collaboration with Stephen Kay and Carole Goble, as well as on the current team [103, 104].

- To be expressive - as a scientific project the goal was to see how close we could come to representing the entire patient history in structured form.

- $\quad$ To be scalable - Larry Weed's PROMIS [128] project with its brilliant touch screen technology for interaction was coming close to its end. We were inspired by the vision, but appalled by the scaling problems of creating an ever accelerating collection of tens of thousands of 'forms' or 'screens' so as to be able to address every possible clinical situation. While Weed himself went on to concentrate on Knowledge Couplers [129, 131, 132], we 
focused on developing intelligent interfaces that could assemble indefinitely many 'forms' from a modest knowledge base.

- To be indefinitely tailorable but to 'fail soft' - to allow developers and users to tailor forms and support for common problems to any required granularity of detail - down to individual users and patients if necessary - but to provide at least some basic interaction for anything that might be said. Perhaps the most surprising thing that it achieved a very broad coverage of medicine with many tends of thousands of potential forms off a compact and manageable knowledge base of under ten thousand assertions.

Meanwhile John Fox and his colleague's work on the Oxford System of Medicine[43] suggested a route to link such systems to broader decision support and knowledge management.

\section{From User Centred Design to Knowledge Representation and Re-use}

\subsection{Everything useful is intractable; everything tractable is useless}

Although it started as a user centred design project, PEN\&PAD rapidly developed major efforts in knowledge engineering and software architecture. The goal became knowledge representation for intelligent interfaces rather than intelligent advice. The obvious approach seemed to be a frame system and a compositional knowledge representation with automatic classification following on along the lines of existing AI toolkits and KL-ONE style knowledge representation systems $[15,137]$.

However, building such a system was potentially daunting. This was only a few years after Brachman and Levesque's discouraging results on the intractability of such 'KL-ONE style' knowledge representation systems [13, 14]. Brachman and Levesque were advocating hybrid systems with radically restricted language for the 'Terminology box' ( 'T-Box'). However, Doyle and Patil's work trying to use such a restricted representation showed such restricted languages to be inadequate for medical terminology [34, 35]. Equally discouraging, results by Touretzky [123], Etherington [36] and eventually Selman [113] made it clear that a complete solution to reasoning with defaults and exceptions in frame based systems was impossible. There appeared to be an impasse: anything computationally tractable was useless; anything useful was computationally intractable.

But we were just building prototypes for user centred design; so we went ahead anyway. Thus was born SMK and the use of a formal representation to drive intelligent medical interfaces, [82, 83]. The surprise was that SMK seemed to work. As we became serious about the development, we faced a further problem: We needed (and had already implemented) several features which were known to be computationally troublesome - notably the fundamental pattern in medical terminology: 'A disease of the part is a kind of a disease of the whole' - e.g. 'a fracture of the shaft of the femur' is a kind of 'a fracture of the femur'. We also soon found ourselves adding a second known problem - making further 'necessary' statements about already defined concepts. Our response was to strip out everything else which was even suspected of being problematic unless we could find evidence for frequent occurrence in medical terminologies. At the same time we declared that anything involving default reasoning was 'extrinsic', borrowing Brachman's metaphor of a 'conceptual coat rack' - the logical classification - on which we hung additional 'extrinsic' knowledge. The result was a highly atypical knowledge representation system - lacking many common constructs but including several which were unusual and computationally expensive but essential to representing medical terminology.

\subsection{GALEN: integration, re-use, and usability}

The GALEN programme on medical terminology emerged from the work on the underpinnings of PEN\&PAD as an attempt to develop 'terminology servers' which would provide rigorous, well defined, compositional terminology[84, 102] based on a frame language with classification. The GRAIL language emerged from SMK, as a suitable frame language to meet that requirement [99]. From its initiation, GALEN was an attempt, on the one hand, to address the problem of terminology in its own right [139], and, on the other hand, to use terminology as the means to re-use information to integrate medical records, decision support, and other clinical systems.

Focusing on re-use was crucial to the development of the GALEN. In PEN\&PAD, there was an intermediate layer between the terminology and user interface, but we were still free to adapt the terminology to change the interface. In GALEN, the terminology had to become fully separate.

With the goal of re-use came also the notion of 'untangling taxonomies' (sometimes put more diplomatically as 'coordinating taxonomies' [95]) - building up each ontology from taxonomies of elementary concepts which form simple disjoint trees. This approach modularises the ontology so that each taxonomy can be changed independently of the others. Use-specific decisions may be embodied in the choice of which abstractions to be defined, but not in the basic taxonomies of elementary concepts.

The task of building the terminology itself proved much greater than any of us imagined at the outset. In retrospect we can discern two distinct phases corresponding roughly to the two projects funded by the European Commission: 
- Establishing the ontology and formalism and demonstrating feasibility by 'druids' in our group in Manchester, which required at least two or three months apprenticeship.

- Developing a scalable terminology based on layered architectures and Intermediate Representations, which reduced training time to do useful work from months to days.

The layered architecture and Intermediate Representation have proved very effective at making the technology accessible [106, 118]. (One might ask why it took us so long to apply what was well known in other knowledge acquisition tasks and even taught in our own classes, but that is another story.)

\subsection{Taming the dilemma of expressiveness and tractability}

In the meantime the underlying technology has begun to catch up with the requirements. Our group spawned specialist groups in description logics and related use of ontologies in hypermedia and bioinformatics [7, 49, 50, 55]. Groups such as the Knowledge Sharing Laboratory at Stanford extended the ideas of developing re-usable ontologies and providing an interchange language amongst the various knowledge representation platforms [39, 41, 52]. In the late 1980s and early 1990s, a sound logical foundation for frame languages with subsumption or 'description logics' was established[6, 12]. In the second half of the 1990s Horrocks and others took those advances and optimised the resulting algorithms to the point of being practically useful[59,60]. This work is now being used increasingly in general applications of information organisation [12, 45, 46], schema integration and as a foundation for knowledge management and meta data - even giving rise to a new proposed standard for an 'Ontology Inference Layer' (originally the 'Ontology Interface Language')[1].

Part-whole and other transitive relations have been extensively studied within the description logic community [4, 5, 88, 111] and elsewhere [86, 136]. The GRAIL representation used in GALEN contains a special construct 'specialisedBy' roughly equivalent to the TRANSFERS-THRO construct in Cyc [71]. Unfortunately, a complete algorithm for this problem is still an unsolved problem[4]. Simple cases can be dealt with by rewriting them into a form which can be thought of as a generalisation of Udo Hahn's triple representation [53, 54]. However, GALEN also uses the same construct to deal with the complex interaction of process, function and causation, and to manage the different types of part-whole relation adapted from Winston [136] and Lenat[71]. Efforts are underway to find a general solution at least to the cases relevant to GALEN.

Nonetheless, current work on description logics offers solid promise of systems which combine inferential power, complete logical semantics, and algorithms which are efficient in practice, even if worst case intractable. The dilemma will remain - expressive power always comes at a cost - but that cost has been reduced. We have learned that 'worst cases' rarely arise in practice, and a 'safe' zone may even be recognisable. Hence the dilemma is less stark[59, 60].

At the same time, the importance of layered architectures to make logical formalisms usable is becoming ever more important. More expressive representations bring with them choices which have non-obvious consequences. In many cases, the best way to express notions is unintuitive, even counter-intuitive. Intuitive user-oriented languages and environments are essential if these powerful systems are ever to be of widespread use. Likewise, the importance of sound ontological structures for both human and machine use has become ever more obvious[105]. There may be no one right way, but there are certainly wrong ways which have unfortunate consequences either for correctness or re-use or both.

\section{Decision support and drug information}

While our group focused on medical records and ontologies, at least four other groups worked on developing re-usable problem solving methodologies - Mark Musen and his colleagues at Stanford with PROTÉGÉ and EON [78-80, 124], Bob Wielinga and his colleagues in Amsterdam with the generic KADS methodology [114, 133, 134], Mario Stefanelli and colleagues in Pavia's work applying KADS and related ideas to medicine in GAMES [38, 112, 126], and John Fox's work on ProForma with its emphasis on safety critical systems [42, 44]. The first three took the distinction between the 'ontology' and the 'problem solving' method as basic, but concentrated more heavily on making the problem solving methods re-usable. The 'ontologies' were relatively simple hierarchies which did not include formal classification algorithms. The idea of combining GALEN's more powerful ontological methods with such re-usable problem solving methods is approaching fruition in UK Department of Health funded PRODIGY and Drug Dictionary programmes.

Early on in GALEN, there had been successful small scale experiments with drug information. Traditional classifications conflate into a single hierarchy chemical structure, physiological action, biochemical action, and clinical indications. A dramatic simplification can be achieved by applying GALEN's techniques to 'untangle' these hierarchies and then using the resulting ontology as a clean framework for 'extrinsic links' to interactions, indications, and contraindications [117]. Almost all special cases and exceptions simply disappear. Over the last two years that effort has been applied on a much larger scale to develop a 'drug ontology' for the UK Department of Health funded PRODIGY and Drug Dictionary projects. 


\section{Terminology and the 'Terminology Wars'}

Terminologies have been both a focus of research and grand challenge in medical informatics [116], and a subject of intense rivalry and controversy, e.g., the studies of coverage carried out by Chute and his colleagues in the early 1990s[23].

In the late 1980s at the start of PEN\&PAD, the idea of compositional terminologies, let alone formal representation, was alien to the prevailing culture International Classification of Diseases, MeSH headings, and Read Codes (now 'Clinical Terms). Despite promising early results [75], the compositional approach was rejected by the National Library of Medicine in favour of the lexical techniques which led to the Unified Medical Language System [72]. However, in 1992, a number of interested groups nonetheless formed the Canon group, issued a manifesto [24, 37] and a series of papers describing their alternative approaches to representing the information in a set of radiology reports [24, 27, 37, 47, 101, 125].

From this base a number of key papers have been developed, many within the framework of the biannual IMIA Working Group 6 meetings, including Cimino's well known paper on Desiderata for clinical systems [25] and Campbell's work on representing SNOMED in Conceptual Graphs and later in description logic[19-21] which eventually gave rise to SNOMED-RT[119]. In the meantime the Read Codes in the UK was undergoing metamorphosis into the Clinical Terms version 3 (CTv3) which provided explicit decompositions but without formal foundations. This work is now being combined with SNOMED-RT to form SNOMED-CT [28].

From the beginning there has been controversy between those arguing for more formality and knowledge representation in medical terminology and those arguing for traditional methods. The claim of one side has always been that the formal methods and ontologies were over-complicated and difficult to understand; the claim of the other that it was the very need for simplicity for clinicians when working with a computer system which required formal sophistication - the 'swans paddle furiously under the water' argument. The dispute is not yet resolved, but the use of a description logic as part of the infrastructure for SNOMED-RT and SNOMED-CT suggests a move in the direction of formality.

Work on practical terminologies has also brought with it the need to confront version and change management more usually addressed in the database community. Change was central to Campbell's proposals for the Convergent Terms Project which eventually became SNOMED-RT[20]. Change management has been explored in more general terms both by Cimino and his colleagues [26] and more recently by Oliver [87]. Full integration of change management and formal techniques remains one of the major challenges facing practical use of formal ontologies.

In the meantime, the central role of medical terminology in medical records now seems to be becoming established , and the aspirations act as the glue amongst applications are beginning to be realised [108].

\section{Language technology}

Simple language generation proved essential to presenting compositional terminologies, at least for GALEN[10, 106, 127, 138]. However, the world of language processing, information retrieval and information extraction have remained remarkably separate from the world of ontologies motivated by logical classification. Swartout[121] and his colleagues managed to reconcile one linguistically oriented ontology (The PenMan Upper Model [8]) with at least parts of the Cyc upper model [71]. However, in general in medical applications, there has been only modest interaction between projects such as GALEN or SNOMED-RT and work such as that by Sager [109, 110] and Friedman[47, 48] on natural language processing or information retrieval such as that by Hersh and colleagues [56, 73].

This is perhaps doubly surprising because one of the important early goals for GALEN was to provide the semantic constraints to extend the work of Rassinoux and Baud in Geneva [3, 9, 93]. This work began well, but never fulfilled its early promise, perhaps because of limits of the coverage of the GALEN ontology at the time. Other work on linking the GALEN ontology to linguistic work revealed major differences in the notions which were considered important by linguists and ontologists motivated by logical classification [22].

There remain real questions concerning when language technology and text retrieval are most appropriate and when logical semantic representation such as GALEN's is needed. The idea of a synthesis of the two is tantalising but so far elusive.

\section{Web Technology, metadata, and integration}

The explosion of the web and web technology seems to me to present three opportunities and a danger. The opportunities are clear - to bring many of the technologies we have been working on into the mainstream, to simplify the infrastructure required, and to generate excitement. The danger is that funding agencies will think that all you need is a few links, a bit of XML, and all the problems will go away. Enormous expectations have been raised, and too few caveats given. 
The aspirations of the Semantic Web [11] and the RDF/RDFs [17, 68] are bringing knowledge representation issues to centre stage. If the explosion of interest in the Ontology Inference Layer (OIL) [1] bears fruit there will be a standard computable ontology formalism widely available.

More broadly, the advent of XML, with its link and pointer mechanisms, provides a mainstream technology capable of supporting more richly interconnected clinical records and supporting a mix of structured and semi-structured data. At the same time, it brings with it the danger that the structured data will stop short of what is needed for decision support. I still believe that 'Marking up is not enough' [97] - but the potential for even loosely structured text to 'reduce muddle' is considerable. It remains to be seen how much of those aspirations can be achieved within the constraints of standards and efforts such as those of HL7, CEN and ISO, but the signs are hopeful.

\section{Synthesis and criteria for success}

The next five years need to see major scaling up of techniques. The challenge of the Web means that things will happen very rapidly. Medical pressures, particularly to reduce errors [67] mean that things need to happen on a large scale.

I would suggest seven issues for the next five years:

- Information capture in clinical medicine. Clinical medicine remains 'information poor'. Little clinical grained information is available in a form which can be used to improve care or reduce 'muddle'. We will know we are succeeding when we can query the data reliably. (Sadly today this is often impossible even in supposedly computerised practices [16].)

- Language processing: Doctors dictate and will continue to dictate. Nurses write and will continue to write. Journals and the annotations to many resource databases are in text and will continue to be in text. The advent of effective speech systems means that more rather than less information will be captured as unstructured, or semistructured, text. We will know that we are succeeding when enough of that information can be made available to support reliably and safely decision support, quality assurance, and access to information sources..

- Re-use, integration, and the harnessing of legacy systems into comprehensive Healthcare Support Systems. Most systems still start 'from scratch', or nearly so. We will know we are succeeding when there are standard resources which many people use for medical record modules, decision support, access to information, and quality assurance, and when those systems can be built separately and put together 'plug-and-play'. There is not even a standard computable source for anatomical information and reasoning - although the Digital Anatomist project has made a major start $[2,74,107]$. The success of the international community around PROTÉGÉ[51] is another pointer that things are moving in the right direction.

- Scaling in complexity and adaptability of systems: Healthcare is big and complicated. The basic problem faced by PEN\&PAD remains unsolved, often not even addressed - how to get the right information and services to the right people at the right time in the right form. Adapting to the clinical problem, task, user, patient, and institutional setting produces a combinatorial explosion which can only be tamed by assembling information dynamically from well organised, coherent parts. We will know we are succeeding when there is a rapid explosion of coherent communicating systems (or 'agents') that really do fit their task and their users.

- Knowledge representation which bridges the different levels of organisation relevant to biomedicine - from molecular biology and genomics through pathophysiology and anatomy to clinical care and population health. If we are to exploit the potential of the exploding knowledge of basic molecular biology, then it must be linked to pathophysiological and clinical information. Ontological techniques intrinsically have the potential to support the interaction of (well constructed) independent ontologies. We will know we have succeeded when people routinely get answers to questions such as 'what genetic markers relevant to the mechanisms of drugs used in this disease taking into account what is already in this patient's medical record and results of gene expression studies.'

- Making sophisticated information available to the public. Masses of public information already exist on the Web, but the means to link it and interpret it are still limited and primitive. Our ability to personalise it is minimal. Public access to their own medical records and information relevant to them in a form they understand is just beginning to appear [122]. We'll know we are succeeding when it becomes the rule rather than the exception.

Knowledge representation and ontologies - more than just 'terminology' - will remain a fulcrum for integration, and the relation between ontologies, medical record models such as the RIM, and decision support will become increasingly critical.

And finally, the greatest challenge: building things that are useful and usable for healthcare professionals and public alike.

\section{Acknowledgements}

The work described has been supported in part by the UK Medical Research Council, the UK Department of Health, and the European Commission. The author wishes to acknowledge the help and support of all members of the 
PEN\&PAD and GALEN teams, with special thanks to Bob Garber who did so much for the team and for PEN\&PAD's user centred design methodology - and who would have done much more had he not died prematurely.

\section{References}

1. The OIL Home Page. www.ontoknowledge.org/oil/

2. Agoncillo AV, Jr MJLV, Rosse C. Influence of the Digital Anatomist Foundational Model on traditional representations of anatomical concepts. Journal of the American Medical Informatics Association 1999(1999 Annual Symposium Issue):2-6.

3. Alpay L, Baud R, A-M R, Wagner J, Lovis C, Scherrer J-R. Artificial Intelligence in Medicine in Europe (AIME93). In:; 1993; Munich; 1993.

4. Artale A, Franconi E, Guarino N. Open problems for part-whole relations. In: International Workshop on Descripition Logics; 1996; Boston, MA; 1996. p. http://www.cs.man.ac.uk/franconi-bin/retrieve/links/franconipapers/part-papers/part-dl96.dvi.gz.

5. Artale A, Franconi E, Pazzi L. Part-whole relations in object-centered systems: An overview. Data and Knowledge Engineering 1996;20:347-383.

6. Baader F, Hollunder B. A terminological knowledge representation system with complete inference algorithm. In: Proceedings of the Workshop on Processing Declarative Knowledge, PDK0-91 (Lecture Notes in Artificial Intelligence \#567); 1991: Springer-Verlag; 1991. p. 67-86.

7. Baker P, Brass A, Bechhover S, Goble C, Paton N, Stevens R. TAMBIS: Transparent Access to Multiple Bioinformatics Information Sources. An Overview. In: Sixth International Conference on Intelligent Systems for Molecular Biology, ISMB 98; 1998; Montreal; 1998. p. 25-34.

8. Bateman JA. Upper modelling: a general organization of knowledge for natural language processing. Penman Development Note: USC/Information Sciences Institute; 1989.

9. Baud R, Lovis C, Alpay L, Rassinoux A-M, Scherrer J-R, Nowlan A, et al. Modelling for Natural Language Understanding. In: Safran C, editor. Seventeenth Annual Symposium on Computer Applications in Medical Care (SCAMC-93); 1993; Washington DC: McGraw Hill; 1993. p. 289-93.

10. Baud RH, Rodrigues J-M, Wagner JC, Rassinoux A-M, Lovis C, Rush P, et al. Validation of concept representation using natural language generation. Journal of the American Medical Informatics Association 1997;(Fall Symposium Supplement):841.

11. Berners Lee T. Semantic Web Road Map. www.w3.org/DesignIssues/Semantic.html

12. Borgida A. Description logics in data management. IEEE Transactions on Knowledge and Data Engineering 1995;7(5):671-682.

13. Brachman R, Fikes R, Levesque H. An essential hybrid reasoning system; knowledge and symbol level accounts of KRYPTON. In: International Joint Conference on Artificial Intelligence (IJCAI-85); 1985: Morgan Kaufmann; 1985. p. 532-539.

14. Brachman R, Levesque H. The tractability of subsumption in frame-based description languages. In: AAAI-84; 1984: Morgan Kaufman; 1984. p. 34-37.

15. Brachman R, Schmolze J. An overview of the KL-ONE knowledge representation system. Cognitive Science 1985;9(2):171-216.

16. Bray J, Majeed A, Kerry S, Rowlands G. Identifying patients with ischaemic heart disease in general practice: cross ssectional study of paper and computerised medical records. British Medical Journal 2000;321:548-050.

17. Brickley D, Guha RV. Resource Description Framework (RDF) Schema Specification 1.0. www.w3.org/TR/2000/CR-rdf-schema-20000327/

18. Brook J, Sheldon M, Rector A. Decision Making in General Practice. London: Macmillan; 1985.

19. Campbell K. Scalable methodologies for distributed development of logic-based convergent medical terminology. Methods of Information in Medicine 1998;37(426-439).

20. Campbell K, Cohn S, Chute C, Rennels G, Shortliffe E. Gálapagos: Computer-based support for evolution of a convergent medical terminology. In: Cimino J, editor. AMIA Fall Symposium; 1996; Washington DC: Hanley and Belfus, Inc; 1996. p. 269-273.

21. Campbell KE, Tuttle MS, Spackman KA. A "lexically-suggested logical closure" metric for medical terminology maturity. Journal of the American Medical Informatics Association 1998(Fall Symposium Special Issue):785-789. 
22. Ceusters W, Rogers J, Consorti F, Rossi-Mori A. Syntactic-semantic tagging as a mediator between linguistic representations and formal modals: an exercise in linking SNOMED to GALEN. Artificial Intelligence in Medicine 1999;15(1):5--24.

23. Chute C, Cohn S, Campbell K, Oliver D, Campbel J. The content coverage of clinical classifications. For The Computer-Based Patient Record Institute's Work Group on Codes \& Structure. Journal of the American Medical Informatics Association 1996;3(3):224-233.

24. Cimino J. Controlled Medical Vocabulary Construction: Methods from the Canon Group. Journal of the American Medical Informatics Association 1994;1(3):296-197.

25. Cimino J. Desiderata for controlled medical vocabularies in the twenty-first century. Methods of Information in Medicine 1998;37(4-5):394-403.

26. Cimino J, Clayton PD. Coping with changing controlled vocabularies. In: Ozboldt JG, editor. Eighteenth Annual Symposium on Computer Applications in Medical Care; 1994; Washington, DC: Hanley \& Belfus, Inc, Philadelphia PA; 1994. p. 135-139.

27. Cimino JC, Hripscak G, Johnson S. Knowledge-based approaches to the maintenance of a large controlled medical terminology. Journal of the American Medical Informatics Association 1994;1(1):35-50.

28. College of American Pathologists. SNOMED Home Page. www.snomed.org

29. Cowell RG, Dawid AP, Lauritzen SL, Spiegelhalter DJ. Probabilistic Networks and Expert Systems. Berlin: Springer-Verlag; 1999.

30. de Dombal F. Surgical diagnosis assited by a computer. Procedings of the Royal Society of London, Series B (Biological Sciences) 1977;184(77):433-440.

31. de Dombal F, Leaper D, Horrocks J, Staniland J, McCann A. Human and computer-aided diagnosis of abdominal pain: further report with emphasis on performance of clinicians. British Medical Journal 1974;1(1974)(904):376380 .

32. de Dombal F, Leaper D, Stanliland J, McCann A, Horrocks J. Computer-aided diagnosis of acute abdominal pain. British Medical Journal 1972;1972(2)(5804):9-13.

33. de Dombal FT. Computers and the surgeon - a matter of decision. Surgery Annual 1979;11:33-57.

34. Doyle J, Patil R. Two dogmas of knowledge representation: language restrictions, taxonomic classification, and the utility of representation services.: Massachusetts Institute of Technology; 1989 1989. Report No.: MIT/LCS/TM-387.

35. Doyle J, Patil R. Two theses of knowledge representation: Language restrictions, taxonomic classification and the utility of representation services. Artificial Intelligence 1991;48:261-297.

36. Etherington D. Formalising nonmonotonic reasoning systems. Artificial Intelligence 1987;31:41-85.

37. Evans DA, Cimino J, Hersh WR, Huff SM, Bell DS, The Canon Group. Position statement: Towards a medical concept representation language. Journal of the American Medical Informatics Association 1994;1(3):207-217.

38. Falasconi S, Lanzola G, Stefanelli M. An ontology-based multi-agent architecture for distributed health-care information systems. Methods sof Information in Medicine 1997;36:20-29.

39. Farquhar A, Fikes R, Pratt W, Rice J. Collaborative Ontology Construction for Information Integration. Technical Report: Knowledge Systems Laboratory, Stanford University; 1996 1995. Report No.: KSL-95-63.

40. Feigenbaum EA, Feldman J, Armer P, editors. Computers and Thought. Cambridge MA: MIT Press; 1968 (reissued 1999).

41. Fikes R, Cutkosky M, Gruber T, Baalen jV. Knowledge sharing technology -- project overview: Stanford University, Knowledge Sharing Laboratory; 1991 November 1991. Report No.: KSL-91-71.

42. Fox J, Das S. Safe and Sound. Cambridge MA: MIT Press; 2000.

43. Fox J, Glowinski A, Gordon C, Hajnal S, O'Neil M. Logic engineering for knowledge engineering; Design and implementation of the Oxford System of Medicine. Artificial Intelligence in Medicine 1990;2(6):323-339.

44. Fox J, Thomson R. Decision support and disease management: a logic engineering approach. IEEE Transactions on Information Technology in Biomedicine 1999;4(2):217-228.

45. Franconi E. A Semantic approach for schema evolution and versioning in object-oriented databases. In: 6th International Conference on Rules and Objects in Databases (DOOD'2000); 2000; London; 2000.

46. Franconi E, Baader F, Sattler U, Vassiliadis P. Multidimensional data models and aggregation. In: Jarke M, Lenzerini M, editors. Fundamentals of Data Warehousing. Berlin: Springer-Verlag; 1999. p. 9-21. 
47. Friedman C, Cimino JJ, Johnson SB. A schema for representing medical language applied to clinical radiology. Journal of the American Medical Informatics Association 1994;1(3):233-248.

48. Friedman C, Hripcsak G, Shagina L, Liu H. Representing information in patient reports using natural language processing and the extensible markup language. Journal of the American Medical Informatics Association 1999;6:76-87.

49. Goble C. TAMBIS Project Summary and documentation. Web Page: University of Manchester; 1996. Report No.: http://www.ca.man.ac.uk/mig/tambis/.

50. Goble C, Haw C, Bechhofer S. Describing and classifying multimedia using the description logic GRAIL. In: Proceedings of the SPIE Conference on Storage and Retrieval of Still Image and Vidio IV, SPIE vol 2670; 1995; San Jose; 1995. p. 132-143.

51. Grosso WE, Eriksson H, Fergerson RW, Gennari JH, Tu SW, Musen MA. Knowledge modelling at the millenium (The design and evolution of Protege-2000). In: Knowledge acqusition workshop (KAW-99); 1999; Banf, Canada; 1999.

52. Gruber TR. Toward Principles for the Design of Ontologies Used for Knowledge Sharing: Knowledge Systems Laboratory, Stanford University; 199323 Aug 1993. Report No.: KSL-93-04.

53. Hahn U, Schulz S, Romacker M. Partonomic reasoning as taxonomic reasoning in medicine. In: Proc. of the 16th National Conf. on Artificial Intelligence \& 11th Innovative Applications of Artificial Intelligence (AAAI99/IAAI-99); 1999; Orlando FL: AAAI Press/MIT Press; 1999. p. 271-276.

54. Hahn U, Schulz S, Romacker M. Part-whole reasoning: a case study in medical ontology engineering. IEEE Intelligent Systems and their Applications 1999;14(5):59-67.

55. Haw D, Goble C, Rector A. GUIDANCE: Making it easy for the user to be an expert. In: Second International Workshop on user Interfaces to Databases; 1994: Springer Verlag; 1994. p. 19-44.

56. Hersh W. Information Retrieval: A Health Care Perspective. Berlin: Springer-Verlag; 1996.

57. Hickam D, Shortliffe E, Bischoff M, Scott A, Jacobs C. The treatment advice of a computer-based cancer chemotherapy protocol advisor. Annals of Internal Medicine 1985;103(6-part 1):928-936.

58. Horan B, Rector A, Sneath E, Goble C, Howkins T, Kay S, Nowlan, WA and Wilson, A. Supporting a Humanly Impossible Task: The Clinical Human-Computer Environment,. In: Diaper D, editor. Interact 90; 1990: Elsevier Science Publishers, B.V.North-Holland; 1990. p. 247-252.

59. Horrocks I. Using an expressive description logic: FaCT or Fiction. In: Cohn AG, Schubert LK, Shapiro SC, editors. Principles of Knowledge Representation and Reasoning: Proceedings of the Sixth International Conference on Knowledge Representation (KR 98); 1998; San Francisco, CA: Morgan Kaufmann; 1998. p. (in press).

60. Horrocks I, Staller U, Tobies S. Practical reasoning for very expressive description logics. Journal of the Interest Group in Pure and Applied Logics (IGPL) 2000;8(3):293-323.

61. Johnson P. What kind of expert should a system be? Journal of Medicine and Philosophy 1983;8:77-97.

62. Johnson P, Duran A, Hassebrock F, Moller J, Pietula M, Feltovich P, et al. Expertise and error in diagnostic reasoning. Cognitive Science 1983;3:91-118.

63. Johnson P, Hassebrock F, Duran A, Moller J. Multimethod study opf clinical judgement. Organisational Behaviour and Human Performance 1983;30:201-230.

64. Kassirer J, Kuipers B, Gorry G. Towards a theory of clinical expertise. American Journal of Medicine 1982;73(2):251-259.

65. Kirby J, Cope N, Souza Ad, Fowler H, Gain R. The PEN\&PAD Data Entry System. In: Brender J, Christensen J, Scherrer J-R, McNair P, editors. Medical Informatics Europe (MIE-96); 1996; Copenhagen: IOS Press; 1996. p. 430-434.

66. Kirby J, Rector AL. The PEN\&PAD Data Entry System: From prototype to practical system. In: Cimino J, editor. AMIA Fall Symposium; 1996; Washington DC: Hanley and Belfus, Inc; 1996. p. 709-713.

67. Kohn LT, Corrigan JM, Donaldson MS, editors. To err is Human: Building a Safer Health System. Washington DC: National Academy Press; 2000.

68. Lassila O, Swick RR. Resource Description Framework (RDF) Model and Syntax Specification. www.w3.org/TR/REC-rdf-syntax/

69. Lauritzen SS, Spiegelhalter DJ. Local computations with probabilities on graphical structures and their application to expert systems. Journal of the Royal Statistical Society, series B 1988;50:253-258. 
70. Ledley R, Lusted L. Reasoning foundations of medical diagnosis. Science 1969;130:9-21.

71. Lenat DB, Guha RV. Building Large Knowledge-Based Systems: Representation and inference in the Cyc Project. Reading, MA: Addison-Wesley; 1989.

72. Lindberg D, Humphreys B, McCray A. The Unified Medical Language System. In: van Bemmel J, editor. 1993 Yearbook of Medical Informatics. Amsterdam: International Medical Informatics Association; 1993. p. 41-53.

73. Malet G, Munoz F, Appleyard R, Hersh W. A model for enhancing internet medical document retrieval with “medical core metadata,". Journal of the American Medical Informatics Association 1999;6:183-208.

74. Mejino JLV, Rosse C. Conceptualization of anatomical spatial entities in the Digital Anatomist Foundation Model. Journal of the American Medical Informatics Association 1999(1999 Annual Symposium Special Issue):112-116.

75. Miller DER. Final Task Report (Task 2) — Unified Medical language System (UMLS) Project: Initial Phase in Developing Representations for Mapping Meedical Knowledge: Internist-I/QMR,HELP and MeSH.: Laboratory for Computational Linguistics, Carnegie Mellon University; 1987 1987. Report No.: CMU-LCL-87-1,.

76. Miller R, Pople H, Myers J. Internist -I, an experimental computer-based diagnostic consultant for general internal medicine. New England Journal Of Medicine 1982;397:468-476.

77. Minsky M, Papert S. Perceptrons: Introduction to Computational Geometry. Cambridge, MA: MIT Press; 1970 (1988).

78. Musen M. Modern architectures for intelligent systems: reusable ontologies and problem-solving methods. Journal of the American Medical Informatics Association 1998(Symposium supplement):46-54.

79. Musen M, Tu S, Das A, Shahar Y. A component-based architecture for automation of protocol-directed therapy. In: Barahona P, Stefanelli M, Wyatt J, editors. Fifth conference on Artificial Intelligence in Medicine Europe (AIME '95); 1995; Pavia, Italy: Springer; 1995. p. 3-16.

80. Musen M, Tu S, Das A, Shahar Y. EON: A component based architecture for automation of protocol-directed therapy. Journal of the American Medical Informatics Association 1996;3:367-383.

81. Nowlan W, Kay S, Rector A, Horan B, Wilson A. PEN\&PAD: A multi-lingual patient care workstation based on a unified representation of the medical record and medical terminology. In: Adlassnig K-P, Grabner G, Bengtsson S, Hansen R, editors. Medical Informatics Europe (MIE-91); 1991; Vienna: Springer-Verlag; 1991. p. 1043-1048.

82. Nowlan W, Rector A. Medical Knowledge Representation and Predictive Data Entry. In: Stefanelli M, Hasman A, Fiesch M, Talmon J, editors. Artificial Intelligence in Medicine Europe (AIME-91); 1991; Maastricht: SpringerVerlag; 1991. p. 105-116.

83. Nowlan W, Rector A, Kay S, Horan B, Wilson A. A Patient Care Workstation Based on a User Centred Design and a Formal Theory of Medical Terminology: PEN\&PAD and the SMK Formalism. In: Clayton P, editor. Fifteenth Annual Symposium on Computer Applications in Medical Care. SCAMC-91; 1991; Washington DC: McGraw-Hill; 1991. p. 855-857.

84. Nowlan W, Rector A, Rush T, Solomon W. From Terminology to Terminology Services. In: 18th Annual Symposium on Computer Applications in Medical Care (SCAMC-94); 1994; Washington, DC; 1994. p. 150-154.

85. Nowlan WA. Clinical workstation: Identifying clinical requirements and understanding clinical information. International Journal of Bio-Medical Computing 1994;34:85-94.

86. Odell JJ. Six different kinds of composition. Journal of Object Oriented Programming 1994;5(8):10-15.

87. Oliver DE, Shahar Y, Shortliffe EH, Musen MA. Representing change in controlled medical vocabularies. Artificial Intelligence in Medicine 1999;15(1):53-76.

88. Padgham L, Lambrix P. A Framework for Part-of Hierarchies in Terminological Logics. In: Sandewall E, Torasso P, editors. KR-94; 1994; 1994. p. 485-96.

89. Patil R, Szolovits P, Schwartz W. Information acquisition in diagnosis. In: Conference of the American Association for Artificial Intelligence (AAAI-82); 1982: Morgan Freeman; 1982. p. 345-348.

90. Patil R, Szolovits P, Schwartz, WB. Modelling knowledge of the patient in acid-base and electrolyte disorders. In: Szolovits P, editor. Artificial Intelligence in Medicine, AAAS Symposium 51. Boulder Colorado: Westview Press; 1982.

91. Pauker S, Gorry G, Kassierer JP, WB S. Towards the simulation of clinical cognition. Taking a present illness by computer. American Journal of Medicine 1976;60(7):981-996.

92. Rasmussen J. Human error and the problem of causality in analysis of accidents. Philsophical Transactions of the Royal Society of London; Series B: Biological Sciences 1990;327(1241):449-460. 
93. Rassinoux A-M. Modeling just the important and relevant concepts in medical language understanding. Methods of Information in Medicine 1998(37):361-372.

94. Reason J, Mycielska K. Absent-Minded? The psychology of mental lapses and everyday errors. Englewood Cliffs, NJ: Prentice Hall; 1982.

95. Rector A. Coordinating taxonomies: Key to re-usable concept representations. In: Barahona P, Stefanelli M, Wyatt J, editors. Fifth conference on Artificial Intelligence in Medicine Europe (AIME '95); 1995; Pavia, Italy: Springer; 1995. p. 17-28.

96. Rector A. The knowledge based medical record - IMMEDIATE-I - a basis for decision support in general practice. In: Lotto Id, Stefanelli M, editors. Inaugural European Conference on Artificial Intelligence in Medicine (AIME85); 1985; Pavia, Italy: North Holland Press; 1985. p. 37-49.

97. Rector A. Marking up is not enough. Methods of Information in Medicine 1993;32(4):272-273.

98. Rector A, Ackerman E. Evaluation rules for sequential diagnosis. Computers and Biomedical Research 1975;8:143-165.

99. Rector A, Bechhofer S, Goble C, Horrocks I, Nowlan W, Solomon W. The GRAIL concept modelling language for medical terminology. Artificial Intelligence in Medicine 1997;9:139-171.

100. Rector A, Brooke J, Sheldon M, PD N. An analysis of uncertainty in British general practice: Implications of a preliminary survey. In: Hunter J, Cookson J, Wyatt J, editors. Second European Conference on Artificial Intelligence in Medicine (AIME-89(; 1989: Springer Verlag; 1989. p. 104-111.

101. Rector A, Glowinski A, Nowlan W, Rossi-Mori A. Medical concept models and medical records: An approach based on GALEN and PEN\&PAD. Journal of the American Medical Informatics Association 1995;2(1):19-35.

102. Rector A, Nowlan W, Glowinski A. Goals for Concept Representation in the GALEN project. In: 17th Annual Symposium on Computer Applications in Medical Care (SCAMC-93); 1993: McGraw Hill; 1993. p. 414-418.

103. Rector A, Nowlan W, Kay S. Foundations for an Electronic Medical Record. Methods of Information in Medicine 1991;30:179-86.

104. Rector A, Nowlan W, Kay S, Goble C, Howkins T. A Framework for Modelling the Electronic Medical Record. Methods of Information in Medicine 1992;32(2):109-119.

105. Rector AL. Clinical Terminology: Why is it so hard? Methods of Information in Medicine 1999;38:239-252.

106. Rector AL, Zanstra PE, Solomon WD, Rogers JE, Baud R, Ceusters W, et al. Reconciling Users' Needs and Formal Requirements: Issues in developing a Re-Usable Ontology for Medicine. IEEE Transactions on Information Technology in BioMedicine 1999;2(4):229-242.

107. Rosse C, Shapiro IG, Brinkley JF. The Digital Anatomist foundational model: Principles for defining and structuring its concept domain. Journal of the American Medical Informatics Association 1998(1998 Fall Symposium Special issue).

108. Rossi Mori A, Consorti F, Galeazzi E. Standrds to support develoment of terminological systems for healthcare telematics. Methods of Information in Medicine 1998;37:551-563.

109. Sager JC. A practical course in terminology processing. Amersterdam/Philadelphia: John Benjamins Publishing Co; 1990.

110. Sager N, Lyman MS, Bucknall C, Nhan NT, Tick LJ. Natural Language Processing and the Representation of Clinical Data. Journal of the American Medical Informatics Society 1994;1(1):142-160.

111. Sattler U. A concept language for an engineering application with part-whole relations. In: International Workshop on Description Logics - DL-95; 1995; Rome; 1995. p. 119-123.

112. Schreiber A, van Heijst G, Lanzola G, Stefanelli M. Knowledge organisation in medical KBS construction. In: Andreassen S, Engelbrecht R, Wyatt J, editors. Fourth Conference on Artificial Intelligence in Medicine Europe; 1993 3-6 October 1993; Munich: IOS Press; 1993. p. 394-405.

113. Selman B, Levesque HJ. The complexity of path-based defeasible inheritance. Artificial Intelligence 1993;62(2):303-340.

114. Shadbolt N. Linking structured knowledge elicitation techniques with CommonKADS knowledge analysis techniques. In: AAAI-94 (submitted to); 1994; 1994.

115. Shortliffe E, Axline S, Buchanan B, Merigan T, Cohen S. An artificial intelligence program to advise physicians regarding antimicrobial therapy. Computers and Biomedical Research 1973;6(6):544-560.

116. Sittig DF. Grand challenges in medical informatics. Journal of the American Medical Informatics Association 1994;1:412-413. 
117. Solomon W, Heathfield H. Conceptual modelling used to represent drug interactions. In: Barahona P, Veloso M, Bryant J, editors. Twelfth International Congress of the European Federation for Medical Informatics, MIE-94; 1994; Lisbon, Portugal; 1994. p. 186-190.

118. Solomon W, Roberts A, Rogers J, Wroe C, Rector A. Having our cake and eating it too: How the GALEN Intermediate Representation reconciles internal complexity with users' requirements for appropriateness and simplicity. Journal of the American Medical Informatics Association 2000(Fall Symposium Special Issue):(In Press).

119. Spackman KA, Campbell KE, Côté RA. SNOMED-RT: A reference Terminology for Health Care. Journal of the American Medical Informatics Association (JAMIA) 1997((Symposium special issue)):640-644.

120. Spiegelhalter D. Computer aided decision making in medicine. British Medical Journal (Clinical Research edition) 1984;289(6445):567-568.

121. Swartout W, Patil R, Knight K, Russ T. Toward distrtibuted use of large-scale ontologies. In: Gaines B, Musen M, editors. Tenth Annual Knowledge Acquisition for Knowledge-Based Systems Workshop (KAW96); 1996; Banf ,Canada; 1996.

122. Szolovits P. Guardian Angels. www.ga.or/ga/

123. Touretzky D. The Mathematics of Inheritance Systems. Los Altos, CA: Morgan Kaufmann; 1986.

124. Tu S, Eriksson H, Gennari J, Shahar Y, Musen M. Ontology-based configuration of problem-solving methods and generation of knowledge-acquisition tools - application of PROTEGE-II to protocol-based decision-support. Artificial Intelligence in Medicine 1995;7:257-289.

125. Tuttle MS. The position of the canon group: A reality check. Journal of the American Medical Informatics Association 1994;1(3):298-299.

126. Vanheijst G, Falasconi S, Abuhanna A, Schreiber G, Stefanelli M. A case-study in ontology library construction. Artificial Intelligence in Medicine 1995;7(3):227-255.

127. Wagner J, Rassinoux A-M, Baud R, Scherrer J-R. Generating noun phrases from a medical knowledge representation. In: Barahona P, Veloso M, Bryant J, editors. Twelfth International Congress of the European Federation for Medical Informatics, MIE-94; 1994; Lisbon, Portugal; 1994. p. 218-223.

128. Weed L. Can the PROMIS be kept? MOBIUS: The Journal of the Alliance for Continuing Medical Education and the Society of Medical College Directors of Continuing Medical Education 1983;3(2):17-24.

129. Weed L. Clinical judgment revisited. Methods of Information in Medicine 1999;38(4-5):279-286.

130. Weed L. Medical Records, Medical Education and Patient Care: The Problem Oriented Medical Record as a Basic Tool. Cleveland: Case Western Reserve Press; 1971.

131. Weed L. New connections between medical knowledge and patient care. British Medical Journal 1997;315(7102):231-235

132. Weed L. Problem-knowledge coupling. Medical Instrumentation 1987;21(5):284-7.

133. Wielinga B, Van de Velde W, Schreiber G, Akkermans H. The KADS Knowledge Modelling Approach. In: The Japanese Knowledge Acquisition Workshop (JKAW '92); 1992; 1992.

134. Wielinga R, van de Velde W, Schreiber G, Akkermans H. Expertise Model Defintion Document: CommonKADS Project Report, University of Amsterdam; 1993.

135. Wilson D, Wilson P, Wilmasley R, Horrocks J, de Dombal F. Diagnosis of acute abdominal pain in the accident and emergency department. British Journal of Surgery 1977;64(4):250-254.

136. Winston M, Chaffin R, Hermann D. A taxonomy of part-whole relations. Cognitive Science 1987;11:417-444.

137. Wood W. The KL-ONE Family. Computers and Mathematics with Applications 1992;23:133-177.

138. Zanstra P, Claassen W, Haring Evd. A natural language generator in Rois. LINKS 1997(2):http://ehm.kun.nl/mi/project/rois/newsltr/Links2/Paper4/paper4.htm.

139. Zanstra P, Rector A, Ceustes W, de Vries Robbé PF. Coding systems and classifications in healthcare: the link to the record. International Journal of Medical Informatics 1998;48(1-3):103-109. 